\title{
A COMUNICAÇÃo NÃO-VERBAL NA ÁREA DA SAÚDE
}

\author{
Non-verbal communication in the health area
}

Ana Paula Ramos ${ }^{(1)}$, Francine Manara Bortagarai ${ }^{(2)}$

\section{RESUMO}

Tema: a comunicação é a base fundamental das relações interpessoais, podendo ser verbal ou não verbal. A comunicação não-verbal completa, contradiz ou substitui a comunicação verbal. Objetivo: realizar uma revisão bibliográfica do uso e do conhecimento da comunicação não-verbal pelos profissionais da saúde. Conclusão: embora os artigos enfatizem a relevância do conhecimento da comunicação não-verbal para melhorar a percepção do paciente como sujeito e humanizar os atendimentos de saúde, diversos profissionais da saúde e estudantes dessa área demonstraram pouco conhecimento acadêmico e profissional sobre essa forma de comunicação.

DESCRITORES: Comunicação não Verbal; Pessoal de Saúde; Humanização da Assistência

\section{INTRODUÇÃO}

O termo comunicar é proveniente do latim communicare - e significa "colocar em comum" ".

Entende-se, a partir dessa definição, que comunicação é o intercâmbio compreensivo de significação por meio de símbolos, havendo ou devendo haver reciprocidade na interpretação da mensagem verbal ou não-verbal ${ }^{1}$. Seja qual for o modo da comunicação, verbal ou não-verbal, ela está sempre presente na cena terapêutica, veiculando conteúdos conscientes e inconscientes, cuja significação está vinculada ao contexto em que ocorre².

De modo especial, a comunicação não-verbal qualifica a interação humana, imprimindo sentimentos, emoções, qualidades e um contexto que permite ao indivíduo não somente perceber e compreender o que significam as palavras, mas também compreender os sentimentos do interlocutor. Mesmo o silêncio é significativo e pode transmitir inúmeras mensagens em determinado contexto ${ }^{3}$.

(1) Fonoaudióloga, Doutora; Professora Adjunto do Curso de Fonoaudiologia da Universidade Federal de Santa Maria UFSM, Santa Maria, RS, Brasil.

(2) Fisioterapeuta do Hospital de Caridade de Santa Maria; Mestranda do Programa de Distúrbios da Comunicação Humana do Curso de Fonoaudiologia da Universidade Federal de Santa Maria - UFSM, Santa Maria, RS, Brasil; Especialista em Fisioterapia Geriátrica e Gerontológica.

Conflito de interesses: inexistente
A comunicação não-verbal abrange cerca de 93\% das possibilidades de expressão, em um contexto de interação social, manifestando-se em $38 \%$ das oportunidades por sinais paralinguísticos, tais como a entonação da voz, os grunhidos, os ruídos vocálicos de hesitação, a pronúncia, a tosse e o suspiro provocados por tensão; e, em 55\%, pelos sinais silenciosos do corpo, como os gestos, o olhar, a postura, a expressão facial, assim como as próprias características físicas, que individualizam o indivíduo dentro de seu contexto específico ${ }^{4}$.

Esses sinais não-verbais podem ser utilizados para complementar, substituir ou contradizer a comunicação verbal e também para demonstrar sentimentos ${ }^{2}$. Em caso de conflito entre a mensagem verbal e a comunicação não-verbal, a mensagem não-verbal prevalecerá ${ }^{\text {. }}$.

Os tipos de sinais não-verbais que podem ser "lidos" durante uma interação de pessoas envolvidas no processo de comunicação são: as ações ou movimentos do corpo, a postura corporal (cinésica), os sinais vocais ou paralinguísticos, o uso do espaço pelos comunicadores (proxêmica), os objetos e adornos utilizados, o tipo de corpo (características físicas) e o momento em que as palavras são ditas ${ }^{2,4,6}$.

Birdwhistell, nos anos 70 , foi considerado o pioneiro na tentativa de compreensão da linguagem do corpo (cinésica), a qual considerou estar contextualizada em seu meio social e cultural ${ }^{4,7}$. Já Hall, introduziu o termo proxêmico, nos anos 80 , a fim de considerar a distância interpessoal como um 
instrumento expressivo na interação não-verbal ${ }^{7}$. Em estudos mais atuais, ressalta-se a importância de se estudar o tema em razão do fato de ser aspecto fundamental para a melhora da interação entre o profissional de saúde e o usuário do sistema de saúde, seja público ou privado $3,7-12$.

Considerando tais pressupostos, este artigo objetiva realizar uma revisão bibliográfica do uso e do conhecimento da comunicação não-verbal pelos profissionais da saúde.

\section{MÉTODO}

Para a realização desta pesquisa foram selecionados apenas artigos brasileiros, publicados nos últimos treze anos, de 1996 a 2009, que enfocassem a importância, a função e as implicações da Comunicação não-verbal para o atendimento em saúde e educação. A consulta ocorreu em periódicos das bases de dados: Scielo, Medline, Periódicos Capes e LILACS.

Os descritores utilizados foram: comunicação não-verbal, cinésica, proxêmica e tacêsica. Utilizando-se a palavra cinésica, foram encontrados quinze artigos, sendo sete destes utilizados; com a palavra proxêmica, foram encontrados seis artigos dos quais quatro foram selecionados, e com a palavra tacêsica, foram encontrados dois artigos, dos quais um foi selecionado para este estudo. $O$ critério de seleção obedeceu à demanda pela abordagem da temática no atendimento em saúde. Os artigos não selecionados abordavam o tema do corpo no âmbito da educação. Do resultado destas buscas, aproveitaram-se também teses e artigos citados em suas referências bibliográficas, o que totaliza 35 artigos, duas teses e três livros que atendiam aos critérios citados, sendo os últimos relevantes ao assunto exposto.

\section{REVISÃO DA LITERATURA}

Em estudos realizados com profissionais da saúde, verifica-se que estes consideram importante o conhecimento da comunicação não-verbal $(\mathrm{CNV})^{7,11-13}$, porque sem tal percepção emocional, os problemas do dia a dia e a rotina podem fazer com que os profissionais toquem sem sentir, olhem sem ver e escutem sem ouvir ${ }^{4}$. Entre os aspectos relevantes para esta análise, encontram-se o uso do espaço (proxêmica), o movimento nesse espaço (cinésica) e a utilização do toque (taxêmica).

O uso do espaço é um meio de comunicação não-verbal e influencia o relacionamento interpessoal $^{4}$. Nomeado como proxêmica, estuda o significado social do espaço e sua estruturação inconsciente $^{2}$. Seu estudo tem contado com um número crescente de investigações, inicialmente, na área da antropologia e psicologia e, hoje, encontram-se vários estudos na área da enfermagem. Estudos realizados com sujeitos queimados ${ }^{14}$, portadores de HIV/AIDS ${ }^{15}$ e laringectomizados ${ }^{16}$ demonstram a importância de se observar as relações espaciais posição e distanciamento, volume de voz e postura corporal - que devem haver entre o profissional de saúde e o sujeito durante a abordagem física deste. Nesses estudos, o volume de voz e o distanciamento dos profissionais frente ao sujeito foram adequados, havendo o predomínio do contato visual durante as interações observadas.

Outro meio de comunicação não-verbal é o toque ou tacésica. $O$ toque deve estar presente em toda assistência, não deve ser condicionado à realização de procedimentos técnicos científicos e deve ter a finalidade de demonstrar carinho, empatia, segurança e proximidade em relação ao sujeito ${ }^{4}$. Tal fato pode ser visualizado em estudos que analisaram esse gesto na díade mãe-filho, este portador de $\mathrm{HIV}^{17}$, e na relação enfermeira e pré-parturientes ${ }^{18}$. Em ambos os estudos, o toque permitiu a intimidade, a segurança e o apoio. Isso também foi verificado em situações na Unidade de Terapia Intensiva, demonstrando o quanto o toque interfere no estado físico e mental do sujeito, podendo gerar segurança e conforto, assim como medo e ansiedade ${ }^{19-21}$.

Abrangendo muitos dos aspectos anteriormente citados, várias pesquisas suscitam a discussão da importância da compreensão da comunicação não-verbal na interação do terapeuta com usuários de atendimentos de saúde mental ${ }^{22}$, em programa de hemodiálise ${ }^{23,24}$, com usuários em estados terminais ${ }^{25}$, com sedados na Unidade de Terapia Intensiva ${ }^{19,26}$, com mulheres que amamentavam pós-parto ${ }^{27}$, com idosos institucionalizados ${ }^{28}$, com sujeitos surdos ${ }^{29}$, com sujeitos oncológicos com ostomia $^{30}$, com crianças autistas ${ }^{31}$ e com sujeito estrangeiro hospitalizado ${ }^{32}$. Em todos os estudos, fica evidente a importância da comunicação nãoverbal na percepção dos sentimentos dos sujeitos, o que tem papel fundamental para o cuidado em saúde. Um exemplo da importância da CNV para a percepção dos sentimentos dos sujeitos foi a investigação acerca da sexualidade de mulheres residentes em uma área rural, que demonstraram desconforto, de modo não-verbal, diante de questionamentos sobre sua vida sexual ${ }^{6}$.

Portanto, a competência em comunicação interpessoal - verbal e não-verbal - é uma habilidade fundamental a ser adquirida, a qual possibilita a excelência do cuidar em saúde ${ }^{33}$. A compreensão da comunicação através de sinais não-verbais é importante principalmente para profissionais cuja 
ação está intimamente relacionada ao corpo e ao movimento ${ }^{7}$, uma vez que contribui de forma relevante para melhorar os relacionamentos, sejam eles pessoais ou profissionais ${ }^{3}$.

Além dessa preocupação relacionada ao sujeito que se comunica, também há pesquisas efetuadas com alunos, futuros profissionais da saúde, sobre suas percepções acerca da comunicação não-verbal emitida pelo sujeito ${ }^{12,34}$ e até mesmo, a percepção que os estudantes possuem de si próprios em termos de comunicação não-verbal e auto-conhecimento corporal ${ }^{35}$. Nesses estudos, foram reveladas as dificuldades dos alunos em interpretarem as manifestações do seu próprio corpo e as ações corporais do sujeito em tratamento.

Os profissionais de saúde não devem esquecer que suas mensagens não são interpretadas apenas pela fala, mas também pela forma como se comportam. Dessa maneira, pode-se tornar a comunicação mais efetiva ao tomar consciência da importância da linguagem corporal, principalmente no que diz respeito à proximidade, à postura, ao toque e ao contato visual ${ }^{4}$.

Quando o profissional se relaciona com o sujeito, primeiramente, aquele deve ouvir o que este fala, em segundo lugar, o que ele não deseja falar e, em terceiro lugar, o que ele não pode falar ${ }^{24}$. Os profissionais de saúde têm a tarefa de interpretar o significado da comunicação não-verbal que o sujeito envia, com a finalidade de estabelecer um plano de cuidados adequado às necessidades singulares do paciente ${ }^{4}$. A passividade dos sujeitos em sua relação com o profissional de saúde pode levá-los a se tornarem apenas um número, um caso clínico, uma ferida ou uma lesão diante de um olhar tecnicista $^{6}$. Por isso, a importância de se considerar a comunicação não-verbal como elo fundamental no processo de cuidado em saúde.

Torna-se impossível o ato do cuidar do enfermeiro, a cura do médico, a reabilitação do fisioterapeuta, a compreensão e o aconselhamento do psicólogo ou qualquer outra ação na assistência ao ser humano, sem haver habilidades de comunicação interpessoal, considerando tal comunicação não apenas um instrumento básico para o relacionamento terapêutico, mas uma competência ou capacidade que deve integrar a formação do profissional da saúde ${ }^{3}$.

Apesar de $65 \%$ de toda a comunicação que ocorre em uma interação ser de caráter não-verbal, sabe-se que em nossa sociedade e, principalmente, em nossas atividades profissionais, a comunicação verbal é a mais difundida e valorizada ${ }^{4,16}$. Isso talvez porque a comunicação não-verbal é mais demorada e requer que os interlocutores estejam atentos para esse tipo de comunicação ${ }^{16}$.
Pode ser caracterizada como uma ocorrência iatrogênica a imprudência do profissional relacionada à má utilização da comunicação não-verbal e a percepção inadequada desta durante a interação com o paciente. Essa ação prejudicial, não intencional, pode ser mais cortante que um bisturi afiado ou mais dolorosa que a dor física. Ela pode trazer sequelas psicológicas ao paciente e influenciar de maneira decisiva no compromisso do mesmo com o terapeuta e na evolução do tratamento8 . $^{2}$

Em pesquisa com estudantes de Medicina, concluiu-se que embora eles considerem importante o conhecimento da comunicação não-verbal para ampliar o vínculo com o paciente e melhorar a compreensão deste, demonstraram não dominar os conceitos, sendo a relação interpessoal com o sujeito predominantemente verbal ${ }^{12}$. Justificando ou não esse resultado, encontra-se o dilema de identidade da medicina, na escolha entre o paradigma da frieza da atual sofisticação tecnológica e impessoal e aquele da interação secular e mágica, da arte do cuidar terapêutico ${ }^{8}$.

Em outra pesquisa com profissionais da Educação Física, Medicina e Psicologia, por meio da análise das entrevistas semiestruturadas, foi possível evidenciar que esses profissionais consideram que o conhecimento e a experienciação dos sinais não-verbais podem torná-los mais habilidosos na percepção e interpretação de estados subjetivos, contribuindo, assim, para melhorar a eficiência e competência profissional ${ }^{7}$. Esses resultados foram também obtidos com a construção de um programa sobre comunicação não-verbal para enfermeiros aplicarem em sujeitos hospitalizados ${ }^{36}$.

Há uma preocupação do fisioterapeuta que atua com sujeitos portadores de deficiência mental e que não oralizam, no que diz respeito à interação com esses sujeitos, uma vez que a formação desses profissionais está mais centrada na promoção do desenvolvimento motor e na reabilitação motora ${ }^{13}$.

Além desses profissionais, o fonoaudiólogo também deve extrapolar os limites dos meros sintomas apresentados pelos sujeitos com dificuldade ou ausência de oralização, buscando o sentido e a interpretação da linguagem manifestada por eles. Sob esta perspectiva, na análise das habilidades verbais e não-verbais efetuada com crianças autistas, com atraso de linguagem, durante uma interação, houve predomínio da linguagem gestual em detrimento dos meios vocais e verbais. Esse fato demonstra que os gestos podem expressar intenções dos sujeitos ${ }^{31}$.

Logo, ressalta-se a importância de formação sobre a CNV junto a profissionais da saúde, já que a linguagem corporal, integrante desta forma de comunicação, ainda não é um tema muito 
discutido na formação profissional11. Nesse sentido, há a necessidade de se superar a visão puramente instrumental nas práticas educativas acerca da gestualidade e dos cuidados com o corpo ${ }^{37}$. Os profissionais da área de saúde, que são realmente preocupados com o ser humano, devem procurar desenvolver meios, instrumentos, técnicas, habilidades, capacidade e competência para oferecer ao paciente a oportunidade de uma existência mais digna, mais compreensiva e menos solitária².

Para a prestação da assistência ao sujeito, é necessário a demonstração de carinho e atenção: ouvir, expressar um olhar atencioso, tocar e reconfortá-lo; tais ações apresentam contribuição essencial à segurança, proteção e auto-estima de uma pessoa ${ }^{1}$. Visto que, ao estabelecer uma boa comunicação com os outros, contribui-se para a diminuição de conflitos e mal-entendidos, tornando as relações mais harmônicas e alcançando os objetivos terapêuticos com mais eficiência e satisfação ${ }^{21}$. Para poder fazer isso, o profissional de saúde necessita dominar os aspectos envolvidos na CNV.

Além da área da saúde, a CNV também tem sido considerada importante na área de marketing e recursos humanos em empresas ${ }^{5}$ e em academias, nas quais os critérios estão cada vez mais relacionados aos canais de comunicação não-verbal como aparência, vestuário, aspecto físico e carisma para a admissão de funcionários. Políticos e executivos também se preocupam com seus gestos, expressões faciais e posturas, para serem mais eficientes ou convincentes em suas comunicações ${ }^{7}$.

Percebe-se, a partir dos artigos revisados, que nos últimos anos, com o desenvolvimento dos estudos voltados para a comunicação, esta se tornou o veículo para os mais belos discursos de humanização em saúde. A ênfase do toque nãotécnico, do olhar não direcionado à busca de patologias, da aproximação sincera e fraterna, quando não amorosa, tem resgatado valores humanos que ficaram em algum lugar no tempo e no espaço da história do homem que, por sua vez, desenvolveu novos modos de adoecer ${ }^{38}$.

Uma vez que a comunicação incide sobre a qualidade da assistência prestada ao sujeito, para alcançar uma comunicação satisfatória é preciso que o profissional da saúde deseje envolver-se e acredite que sua presença é tão importante quanto a realização de procedimentos técnicos ${ }^{1,2-4,7,11}$. Para tanto, deve assumir uma visão de saúde mais holística, na qual não há um divórcio entre corpo e mente 38,39 .

Ao longo da história, o corpo foi associado à alma (Platão, Demócrato, Santo Agostinho), limitado a fluidos corporais (Hipócrates), locus dos defeitos e pecados (Idade Média) e seccionado da sua relação com a mente, sendo a religião e a filosofia detentora do estudo da mente e o estudo do corpo, visto como uma máquina, objeto de estudo da medicina (Descartes). O rompimento dessa visão mecanicista do corpo do homem foi introduzido com a teoria psicanalítica de Freud e, mais tarde, com a introdução do termo psicossomático por Heinroth e Alexander, o que denota uma visão mais holística devido à inseparabilidade dos aspectos biológicos e psicológicos ${ }^{40}$.

$\mathrm{Na}$ atualidade, o corpo mantém um status de beleza calcado no estereótipo musculoso para os homens e magro para as mulheres ${ }^{35}$. Ele é julgado na superficialidade, pelo seu exterior, seja nas relações interpessoais quanto em uma avaliação nas diferentes áreas da saúde. A leitura do seu silêncio e a grandeza de suas manifestações e interpretações são pouco conhecidas pelos profissionais da saúde ${ }^{2,47,71,12}$. Esse fato pode ser observado a partir dos resultados desta revisão.

Retomando a perspectiva holística, a subjetividade do usuário é considerada e a comunicação não-verbal passa a ter um papel importante na cena terapêutica, como elemento de acesso, assim como a linguagem verbal, aos valores culturais e pessoais do paciente ${ }^{4}$. Tais aspectos são considerados relevantes no estabelecimento de uma boa relação interpessoal, com a finalidade de humanizar 0 atendimento em saúde ${ }^{40}$. Parece, no entanto, um paradoxo falar em humanização do atendimento de saúde, visto que ninguém racionalmente pensaria que tal atendimento pudesse ser desumano.

A realidade demonstra que o curso da especialização em saúde e a forma como ela foi conduzida a partir de um modelo de ensino médico baseado na racionalização científica, com a crença naquilo que é visível, palpável, que possa ser dissecado e delimitado numericamente, produziu uma fragmentação do sujeito, que recebe, nessa concepção, a denominação de paciente ${ }^{40}$. Por isso, o pouco interesse em observar as manifestações não-verbais desse sujeito ${ }^{4,7,8,10} \mathrm{e}$ a ausência de reflexão sobre o papel do corpo do terapeuta ${ }^{12,33-35}$.

A fragilidade do ensino e da aprendizagem da CNV demonstrada pelos graduandos da saúde demonstra a importância de disciplinas voltadas para o conhecimento teórico e prático desse tema ${ }^{12,34}$. Os currículos das escolas dos futuros profissionais da saúde valorizam pouco os conteúdos relacionados à humanização da assistência, enquanto há hipervalorização dos conteúdos técnicos relacionados exclusivamente aos aspectos biológicos do ser humano $0^{12,35}$. No entanto, à medida que se amplia a percepção do futuro profissional da saúde através da reflexão do fazer e do entender o usuário, surgem novos elementos para a melhoria 
da qualidade dos serviços de saúde através da competência e excelência do atendimento ${ }^{7}$ e a CNV é uma das temáticas relevantes $n$ esse processo de ampliação.

\section{CONCLUSÃO}

A aprendizagem da comunicação não-verbal não só é possível, como necessária, em vista da sua importância para as interações intersubjetivas, em todos os níveis de nossa vida: pessoal, social, profissional dentre outros e permite a ampliação da atuação em saúde para uma perspectiva promocional.

Durante a busca de artigos e estudos referentes à temática aqui abordada, a área que mais concentrou pesquisas brasileiras da CNV foi a de Enfermagem, confirmando a "supremacia" do cuidar e a busca da qualidade no atendimento.
A promoção de uma assistência holística que envolva as necessidades bio-psico-sócio-espirituais e emocionais deve passar por um processo comunicativo eficaz entre terapeuta-sujeito.

Diferente das demais áreas, a Enfermagem, desde a década de 50, vem desenvolvendo inúmeros estudos sobre a arte do cuidar e seu aperfeiçoamento. Dentre os cuidados pesquisados, está a interpretação da comunicação não-verbal do sujeito.

Trabalhos sobre a CNV, realizados por fisioterapeutas e fonoaudiólogos, foram escassos. Logo, sugere-se que o termo "cuidar" seja aderido pelos demais profissionais atuantes na saúde, obtendo alcance semelhante ao que este tem na enfermagem. Sugere-se, também, que novos estudos sejam realizados pelos profissionais que trabalham não somente com a patologia, mas com sujeitos que apresentam queixas, desejos e demandas.

\begin{abstract}
Background: communication is the fundamental basis of interpersonal relationship and it can be verbal or non-verbal. Non-verbal communication completes, contradicts or substitutes verbal communication, improving the perception of the patient as a subject and humanizing health services.

Purpose: to conduct a bibliographical review on the use and knowledge of non-verbal communication by health professionals. Conclusion: although the articles emphasize the relevance concerning the knowledge of non-verbal communication in order to improve the perception of the patient as a subject and humanizing health services, many health professionals and students from this area showed little academic and professional knowledge on this communication mean.
\end{abstract}

KEYWORDS: Nonverbal Communication; Health Personnel; Humanization of Assistance

\section{REFERÊNCIAS}

1. Oriá MOB, Moraes LMP, Victor JF. A comunicação como instrumento do enfermeiro para o cuidado emocional com o cliente hospitalizado. Rev. Eletrônica Enfermagem. 2004; 11(6):292-5.

2. Stefanelli MC. Comunicação com o paciente: teoria e ensino. São Paulo: Robe;1993.

3. Araújo MMT, Silva MJP, Puggina ACG. A comunicação não-verbal enquanto fator iatrogênico. Rev. Esc. Enferm. USP. 2007; 41(3):419-25.

4. Silva MJP. Comunicação tem remédio: a comunicação nas relações interpessoais em saúde. 3 ed. São Paulo: Loyola; 2002.

5. Schelles S. A importância da linguagem não-verbal nas relações de liderança nas organizações. Rev. Esfera. 2008; ND(1):1-8.
6. Ressel LB, Silva MJP. Reflexões sobre a sexualidade velada no silêncio dos corpos. Rev. Esc. Enf. USP. 2001; 35 (2): 50-154.

7. Mesquita RM. Comunicação Não-verbal: relevância na atuação profissional. Rev.paul.Educ. Fis. 1997; 11(2):155-63.

8. Pericardis AAM. Comunicação iatrogênica na cancerologia. Rev. Soc Bras Cancerol. 1999; 8(2):11-3.

9. Siqueira $A B$, Filipini $R$, Posso $M B$, Fiorano AM, Gonçalves AS. Relacionamento enfermeiro, paciente e família: fatores comportamentais associados à qualidade de assistência. Arq. Med. ABC. 2006; 2 (31):73-7.

10. Tavares FM. Reflexões acerca da iatrogenia e educação médica. Rev. bras. educ. med.2007; 31(2):419-25. 
11. Silva LMG, Brasil, VV, Guimarães HC, Savonitti $\mathrm{BH}$, Silva MJP. Comunicação não-verbal: reflexões a cerca da linguagem corporal. Rev. latino-am. enfermagem. 2000; 8(4):52-8.

12. Moneia ACL. A importância e a compreensão verbal e não verbal sob a ótica do graduando de medicina [dissertação]. São Paulo (SP): Escola de Enfermagem, Universidade de São Paulo; 2002.

13. Ribeiro J, Moraes MVM, Beltrame TS. Tipo de atividade e relação interpessoal estabelecida entre fisioterapeuta e criança com paralisia cerebral no contexto de intervenção fisioterapeutica. Dynamis revista tecno-científica. 2008; 14(1):89-95.

14. Silva MF, Silva MJP, Menezes MAJ. Análise dos fatores proxêmicos na interação dos profissionais de saúde com os pacientes queimados. Rev. Paul. Enferm. 2006; 25(1):4-10.

15. Galvão MTG, Paiva SS, Sawada NO, Pagliuca LMF. Análise da comunicação proxêmica com portadores de HIV/AIDS. Rev. latinoam. Enferm. 2006; 14(4): 491-6.

16. Sawada NO, Zago MMF, Galvão CM, Ferreira $\mathrm{E}$, Barichello $\mathrm{E}$. Análise dos fatores proxêmicos na comunicação com o paciente laringectomizado. Rev. latinoam. Enferm. 2000; 8(4):72-8.

17. Galvão MTG, Costa E, Lima ICV, Paiva SS, Almeida PC, Pagliuca LMF. Comunicação não-verbal entre mãe e filho na vigência do HIV/AIDS. Esc. Anna Nery Rev. Enferm. 2009; 13(4):780-5.

18. Pinto CMS, Rocha EA, Silva MJP. O toque como elemento da comunicação parturiente/ enfermeiro obstetra durante a assistência no pré-parto. Nurse 2002; 47:31-4.

19. Zinn GR, Silva MJP, Telles SCR. Comunicar-se com o paciente sedado: vivência de quem cuida. Rev Latino-Am. Enfermagem. 2003; 11(3):326-32.

20. Barlem ELD, Rosenhein DPN, Lunardi VL, Lunardi Filho WD. Comunicação como instrumento de humanização do cuidado de enfermagem: experiências em unidade de terapia intensiva. Rev. Eletr. Enf. 2008; 10(4):1041-9.

21. Gala MF, Telles SCR, Silva MJP. Ocorrência e significado do toque entre profissionais de enfermagem e pacientes de uma UTI e Unidade Semi-intensiva cirúrgica. Rev. Esc. Enferm.USP. 2003; 37(1):52-61.

22. Castro RBR, Silva MJP. A comunicação não-verbal nas interações enfermeiro-usuário em atendimentos de saúde mental. Rev. latino-am. Enfermagem. 2001; 9(1):80-7.
23. Gullo ABM, Lima AFC, Silva MJP. Reflexões sobre comunicações na assistência de enfermagem ao paciente renal crônico. Rev. Esc. Enferm. USP. 2000; 34 (2):209-12.

24. Dias MAS, Vieira NFC. A comunicação como instrumento na promoção de saúde na clínica dialítica. Rev. Bras. de Enferm. 2008; 61(1):71-7.

25. Araújo MMT, Silva MJP. A comunicação com o paciente em cuidados paliativos: valorizando a alegria e o otimismo. Rev Esc Enferm USP. 2007; 41(4):668-74.

26. Ordahi LFB, Paoilha MIC, Souza LNA. Comunicação entre a enfermagem e os clientes impossibilitados de comunicação verbal. Rev. Latino-Am. Enfermagem. 2007; 15(5):965-72.

27. Leite AM, Silva IA, Scochi CGS. Comunicação não-verbal: uma contribuição para o aleitamento em amamentação. Rev. Latino-Am. Enfermagem. 2004; 12 (2):258-64.

28. Machado ACA, Brêtas ACP. Comunicação não-verbal de idosos frente ao processo de dor. Rev. Bras. Enferm. 2006; 59 (2):129-33.

29. Chaveiro N, Porto CC, Barbosa MA. Relação do paciente surdo com o médico. Rev. Bras. Otorrinolaringol. 2009; 75(1):147-50.

30. Paegle SO, Silva MJP. Análise da comunicação não-verbal de pessoas portadoras de ostomia por câncer de intestino em grupo focal. Rev. Latino-Am. Enfermagem. 2005; 13(1):46-51.

31. Campelo LP, Lucena JA, Lima CN, Araújo, AMM, Viana LGO, Veloso MML, Correia PIF, Muniz LF. Autismo: um estudo de habilidades comunicativas em crianças. Rev. CEFAC. 2009; 11(4):598-606.

32. Chubaci, RYS, Merighi MAB. A comunicação no processo da hospitalização do imigrante japonês. Rev. Latino-Am. Enfermagem. 2002; 10(6):805-12.

33. Braga EM, Silva MJP. Comunicação competente - visão de enfermeiras especialistas em comunicação. Acta Paul. Emferm. 2007; 20(4):410-4.

34. Jesus MCP, Cunha MHF. Utilização dos conhecimentos sobre comunicação por alunos de graduação em enfermagem. Rev Latino-am Enferm. 2008; 6(1):15-25.

35. Narciso ALG. Corpos em evidência: reflexões sobre a exposição corporal no curso de fisioterapia [dissertação]. Sorocaba (SP): Universidade de Sorocaba; 2006.

36. Silva MJP. Aspectos gerais da construção de um programa sobre comunicação não-verbal para enfermeiros. Rev. Latino-Am. Enfermagem. 1996; 4(2):1-8. 
37. Mendes MI, Nóbrega TP. Corpo, natureza e cultura: contribuições para a educação. Revista Brasileira de Educação. Campinas. 2004; 5(1):19-25.

38. Penha RM; Silva MJP. Do Sensível ao Inteligível: novos rumos comunicacionais em saúde por meio do estudo da Teoria Quântica. Rev. esc. enferm. USP. 2009; 43(1):13-22.
39. Helman CG. Cultura, saúde e doença. Porto Alegre : Artmed, 2003.

40. Castro MG, Andrade TMR, Muller MC. Conceito mente e corpo através da história. Rev.Psicologia em Estudo. 2006; 11(1):39-43.

Endereço para correspondência:

Francine Manara Bortagarai

R. Appel, 800, apto 210

Santa Maria - RS - Brasil

CEP: 97030-015

E-mail: fbortagarai@ hotmail.com 